\title{
A scintigraphic study of local oesophageal bolus transit: differences between patients with Barrett's oesophagus and healthy controls
}

\author{
A W Stier, H J Stein, H-D Allescher, M Feith, M Schwaiger
}

See end of article for authors' affiliations

.....................

Correspondence to: Dr A Stier, Chirurgische Klinik der Ernst Moritz

Arndt Universität, Friedrich Löffler Strasse 23b,

D-17487 Greifswald,

Germany;

stier@uni-greifswald.de

Accepted for publication 9 April 2001
Background: In Barrett's patients, functional disorders of oesophageal motility are currently measured by oesophageal manometry. Yet abnormalities of oesophageal volume transport in the critical regions of the upper oesophageal sphincter (UOS) and lower oesophageal sphincter (LOS) cannot be determined using these methods.

Aims: To further characterise the activity of the sphincter regions, we developed a quantitative method for differentiation of oesophageal volume transport in Barrett's patients and healthy controls.

Methods: We used a new technique of processing scintigraphic images, with data analysis based on a new concept of relative local transit time. Twelve patients with Barrett's oesophagus and 11 healthy volunteers were examined using alimentary scintigraphy after a semisolid test meal in a multiple swallow test. In individual scintigraphic images of five swallows we studied: (1) overall oesophageal clearance and (2) the topographic profile of the relative local transit time obtained by image conversion to a two dimensional line graph. This profile was reconstructed by assembling constituent Gauss bands, allocating their integrals to five oesophageal regions according to their band position.

Results: (1) Overall oesophageal clearance was not significantly different between the two groups. (2) In comparison with healthy volunteers, relative regional transit times of all 12 Barrett's patients were significantly increased in the hypopharyngeal region and decreased in the region of the distal oesophagus. The extent of the decrease in the region of the distal oesophagus showed a close correlation with the length of Barrett's metaplasia.

Conclusion: Improvement in image processing allows alimentary scintigraphy to describe different regional patterns of oesophageal volume transport. Local oesophageal bolus transit is markedly abnormal in Barrett's patients without alteration in clearance. The presence of metaplasia itself implies a negative impact on both sphincter functions. These findings substantiate the diagnostic value of refined oesophageal scintigraphy.
D isturbance in oesophageal motility, as demonstrated by manometry, is a well established finding in Barrett's patients. ${ }^{1}$ In addition to mechanical incompetence of the lower oesophageal sphincter (LOS), lower amplitudes of peristaltic waves in the tubular portion of the oesophagus have been reported in most but not all Barrett's patients. A decrease in amplitude and an increase in the duration of contraction has been found to be approximately related to the extent of Barrett's metaplasia. ${ }^{2}$ In principle, determination of volume transport may compliment or even serve as an alternative to manometry because physiological or pathological transport mechanisms cannot be directly observed or predicted from manometry alone; it is probable that clearance is related to peristaltic pressure but not bolus speed. This is due to the fact that peristaltic pressure, as determined by manometry, is only one of several factors that influence oesophageal transport. Other factors such as propulsive force ${ }^{3}$ or longitudinal shortening, ${ }^{45}$ which are essential for transport, are not measurable by manometry. Two standard techniques can be used to measure volume transport, scintigraphy ${ }^{6}$ and radiography. ${ }^{7}$ However, only scintigraphy quantitatively measures bolus transport.

Until now, scintigraphic studies of oesophageal transport have rarely been performed in Barrett's disease and their results have been inconclusive. A typical example of such a study is one in which five Barrett's patients and 83 other patients suffering from five different oesophageal diseases were studied using non-parametric grading of oesophageal clearance. The six different disorders could not be dis- tinguished from one another. ${ }^{8}$ Another study of Barrett's disease investigated time activity curves in 27 patients: $52 \%$ of these patients had abnormal transit times $(90 \%$ of the oesophagus emptied in more than 12 seconds) and $48 \%$ had abnormal degrees of emptying (less than $90 \%$ in 30 seconds). No correlation between the functional studies and the length of Barrett's epithelium was found. ${ }^{9}$ These studies determined only overall clearance of the oesophagus. However, the regions of the oesophagus are functionally different. Correspondingly, the complex factors controlling bolus transport vary within the different oesophageal segments ${ }^{10}$ and may cause regionally specific variations in pathogenetic mechanisms. Therefore, a quantitative description of the transit of bolus mass must be closely related to oesophageal anatomy. The only technique which can measure the kinetics of the transport of the bolus mass quantitatively is scintigraphy. This means that scintigraphy is indispensable for the study of oesophageal transit patterns. Another positive aspect of this technique is the fact that the examination itself is rapid and simple to perform, making it highly acceptable for patients. For the realisation of this concept of "locoregional transit times", development of new techniques for evaluation and representation of the information contained in the scintigraphic images is necessary; the

Abbreviations: LOS, lower oesophageal sphincter; UOS, upper oesophageal sphincter. 


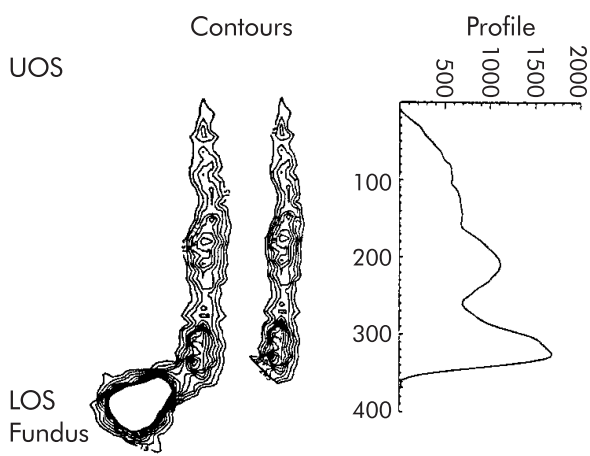

Figure 1 Multicontour plots and profile of the three dimensional swallow of a control subject based on summation of all consecutive images (total eight) taken during this single swallow. The two contours demonstrate the effect of the mask for cutting off the fundus at the lower oesophageal sphincter (LOS). The profile has the same length, expressed in terms of lines, as the array of the contour.

required spatiotemporal pattern of oesophageal bolus transport must allow extraction of quantitative data that can be useful as pathological indices.

The aims of the present study were: (1) to develop image processing techniques for visualisation of the spatiotemporal pattern of oesophageal bolus transit and methods for quantitative data evaluation allowing comparative studies; (2) to compare the techniques developed with published tools; and (3) to apply these tools to the investigation of the pathophysiology of oesophageal bolus transport in Barrett's patients by comparison with normal transport in controls, thus identifying pathognostic indices.

\section{METHODS}

\section{Subjects}

Two groups of subjects were studied: 11 healthy controls (eight males and three females; mean age 47.3 years (range 28-63)) without symptoms or a history of gastrointestinal disease were compared with 12 patients with Barrett's oesophagus (nine males, three females; mean age 51 (range 27-70)) who had symptoms of gastro-oesophageal reflux disease. The presence of Barrett's epithelium was confirmed by histological documentation of intestinal metaplasia on oesophageal biopsies. The length of Barrett's metaplasia, as determined by endoscopy, ranged from 2 to $9 \mathrm{~cm}$. None of the patients or volunteers had previous upper gastrointestinal surgery. On endoscopy, the presence of a sliding hiatus hernia was documented in 11/12 patients with Barrett's oesophagus and in none of the control subjects. Medication that may influence bolus transport was discontinued at least seven days prior to the investigation. The study protocol was approved by the medical ethics committee of the Technische Universitaet Muenchen.

\section{Scintigraphy}

Patients were seated upright with their back to the camera (posterior projection). The upper border of the gamma camera (Picker) was positioned in a horizontal plane, $2 \mathrm{~cm}$ above the incisura jugularis sterni. Thus the field of view included the oesophagus, extending from its lower cervical portion to the cardia, and the fundus. To avoid inclusion of a hiatal hernia in the regional analysis of transit times, the positions of the LOS and upper oesophageal sphincter (UOS) were determined by contour plots. In all subjects, both sphincter regions could be clearly visualised (fig 1). The distance between the UOS and LOS was defined as $100 \%$ oesophageal length. All regional analyses were performed in relation to the UOS and LOS to prevent bias in data interpretation due to a shorter extent of the oesophagus in patients with a hiatal hernia.
The semisolid test meal, a purée (instant powder for children; Aurora, Weinheim, Germany), was labelled with 80 MBq 99m technetium DTPA (effective dose $1.8 \mathrm{mSv}$ ). After a two hour fasting period, subjects were given five consecutive portions ( $10 \mathrm{ml}$ each) of the test meal with a syringe at about 30 second intervals. Continuous recording was started $30 \mathrm{sec}-$ onds before ingestion of the first portion. A total of 210 sequential images were sampled in a frame mode $(0.8$ $\mathrm{s} /$ frame). All images were acquired on a $64 \times 64$ matrix and stored in a dedicated computer (Siemens, Munich, Germany). The scintigraphic data were formatted and transferred for image processing and analysis to an IBM compatible personal computer.

\section{Image processing}

The concept of relative local transit times was realised by construction of a quantitative spatiotemporal pattern of bolus transit. Profiles of bolus structures represent this pattern, as illustrated by the diagram in fig 2 . These profiles are obtained by summation of the pixel counts of the image rows. Hence the topographical lateral $\mathrm{x}$ coordinate of the image is compressed and the original three dimensions are reduced to the two dimensions of a line graph which further serves for quantitative data evaluation (see below). The profile of one image visualises the time dependent bolus distribution during the time of image acquisition ( 0.8 seconds) while the profiles of the sum of all images of one swallow is the transitory distribution of the bolus during its oesophageal transport. The y coordinate of the profile is proportional to the local time the bolus needs to transit consecutive sections of the oesophagus. This relationship can be understood by considering the flow of a scintillating fluid in a tube. The count rate measured at a certain cross section is inversely proportional to the velocity of flow and accordingly proportional to transit time. In a tube consisting of segments differing in their cross sections, transit times of the fluid in the different segments are proportional to their cross sections (equation of continuity of flux). The latter relation also applies to a distensible tube, such as the oesophagus, which has a variable diameter.

In detail, the profile was obtained by summation of the pixel counts of rows of the three dimensional matrix of the image sum of one swallow or of five swallows. Before row summation, a mask was interactively formed which cuts off all of the image matrix outside the oesophagus, as illustrated with the help of multicontour plots (fig l). This mask avoids counting of pixels of the fundus which overlap pixels of the lower end of the oesophagus. Moreover, from these multicontour plots the length of the profile and the length of the oesophagus can be shown to be equal, as also demonstrated in fig l. Relative local transit times are obtained by standardisation: the sum of each row is divided by the average of the sums of all rows. Furthermore, row summation of the total of all images of five swallows averages the local kinetics of bolus transit of these swallows. This multiple swallow technique allows for intraindividual variation in swallows by minimising the influence of single aberrant swallows. A major advantage of this image addition technique is the considerable increase in the signal to noise ratio.

\section{Multicontur plot}

This procedure draws the data stored in a pixel based array as a topographical distribution of the bolus in the oesophagus. As used for geographical mapping, lines are generated connecting pixels containing the same number of counts. Stepwise addition of these numbers produces multiple contours. The number and size of the steps are chosen for optimal visualisation of the data. Spacing and number of lines allow an easy way of reading the quantitative topographical distribution of the bolus.

These image processing tools were developed with the help of IDL (Research Systems Inc.). 


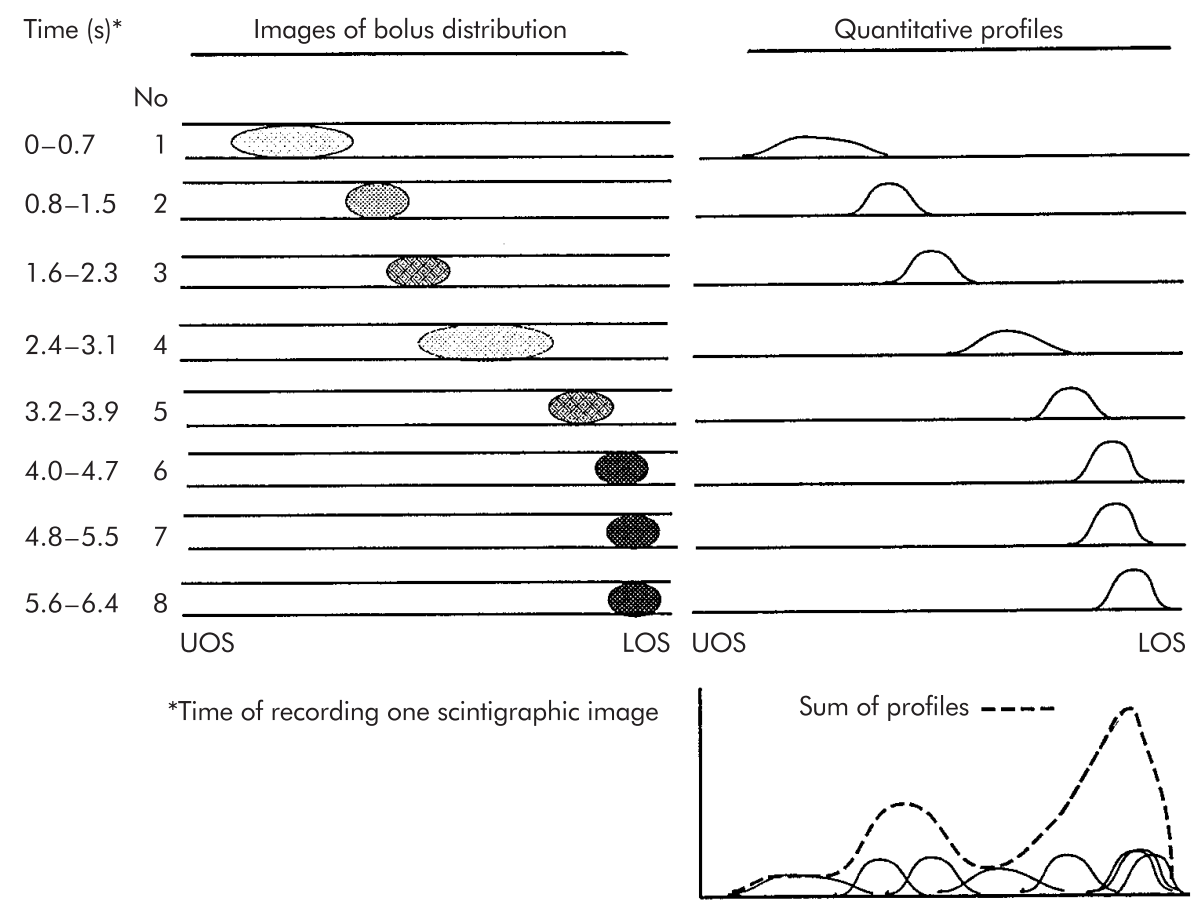

Figure 2 Diagrammatic representation of the definition of local transit times. The eight frames of oesophageal distribution and progression of the bolus of a single swallow (swallow No 3) are shown. Time of image acquisition is 0.8 seconds. Three velocities of bolus progression have been chosen: velocities in images 1 and 4 are greater than those in images 2,3, and 5 which are greater than those of images 6-8. This order corresponds to an increase in count rates on scintigraphic imaging, as shown by the increasing shading of the boluses. The height of the profiles is proportional to this intensity and inversely proportional to the velocity of bolus progression and correspondingly proportional to the local transit time of the bolus mass. Accordingly, the $y$ axis values of the sum of profiles (see graph at the lower right) are proportional to local transit time. It is important to note that the profile of an image acquired in 6.4 seconds, which records the whole oesophageal bolus passage, has the same shape as this sum of eight profiles of images each acquired in 0.8 seconds. LOS, lower oesophageal sphincter; UOS, upper oesophageal sphincter.

\section{Data analysis}

The profile as a two dimensional coordinate system facilitates the definition and evaluation of the mathematical relationship between parameters. For the purpose of an interindividual comparison, both coordinates of the profile have to be standardised. Regarding the y coordinate, a relative local transit time was defined by division of the pixel counts of individual rows by the average of counts in all rows along the oesophagus. The x coordinate, the length of the oesophagus, was determined from the number of column lines of the contour plot, as shown for the sum of images of one swallow in fig 2; this number was set as $100 \%$ length. Fitting of Gaussian bands for normal distribution to the profile was found to be an excellent technique for quantitative evaluation of transit
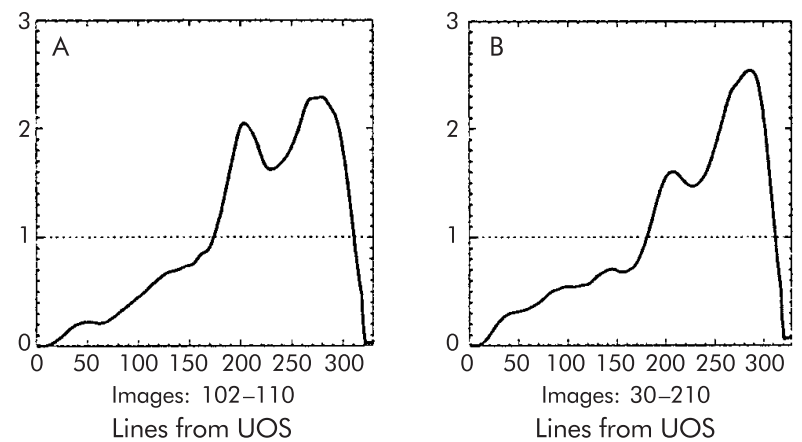

Figure 3 (A) Profile of relative local transit times of the third swallow. (B) Averaged profile of relative local transit times of five swallows of a control subject. The y coordinates of each curve were normalised by dividing by the average y values. The broken line represents this average. UOS, upper oesophageal sphincter. kinetics. Usually the profile could be reconstructed by fitting about eight bands using the program Origine (Microcal Software Inc.). The integrals of individual bands were expressed as fractions of the sum of all bands and allocated to five topographical regions (segments of equal length) in the oesophagus, according to their band position. The resulting parameters were named relative regional transit times. The techniques used in this study for image processing and quantitative evaluation of the scintigraphic images of oesophageal bolus transit differ from the methods used in any previous study. ${ }^{11-13}$ Oesophageal clearance was determined by the multiple swallow version of the condensed image technique. ${ }^{13}$

\section{Statistics}

$\mathrm{T}$ tests and linear regression were performed with tools of Excel, version 7 (Microsoft).

\section{RESULTS}

The spatiotemporal pattern of relative local transit times

A qualitatively similar spatiotemporal pattern was observed in all subjects, in patients as well as controls. The spatiotemporal profile of the third swallow of a control subject shows the "relative local times" that the bolus needs to pass through consecutive oesophageal segments (fig 3A). The cervical portion of the oesophagus has by far the shortest relative local transit time. Transit is about five times faster than the average transit. Near the mid portion of the oesophagus, the region of transition of striated to smooth muscle, the relative local transit time begins to exceed the average overall local transit time and reaches a maximum value at two sites, one at the transit of the bolus through a plane located at $60 \%$ of the oesophageal length distal from the UOS, and one immediately proximal to 


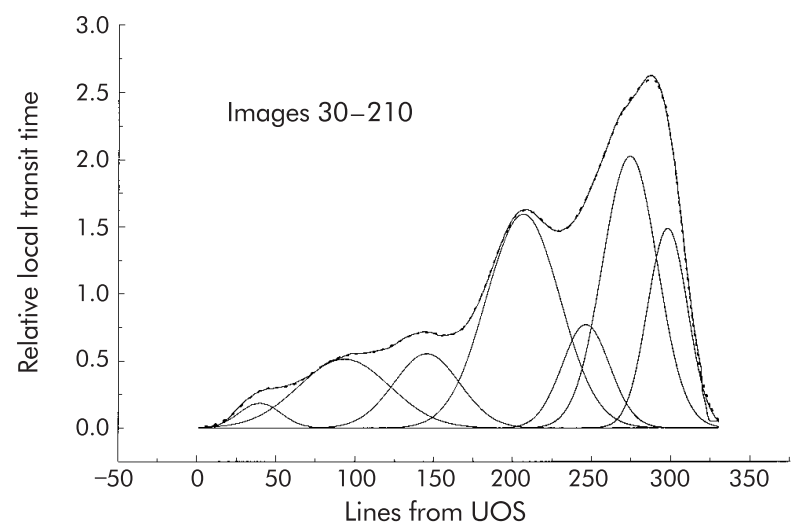

Figure 4 Curve fitting of the profile including all images of five swallows in a control subject using Gaussian bands. Note that the curve of the sum of all fitted integrals precisely follows the original curves, as shown by the broken line. For other conditions see fig 3B UOS, upper oesophageal sphincter.

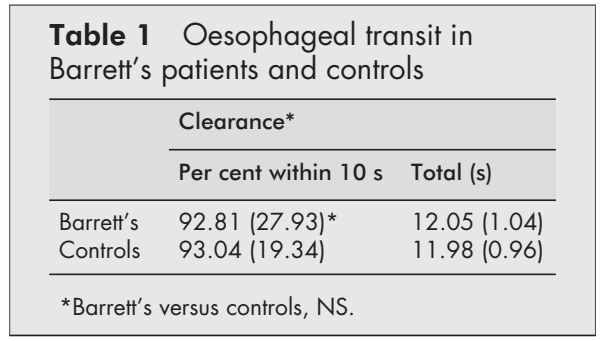

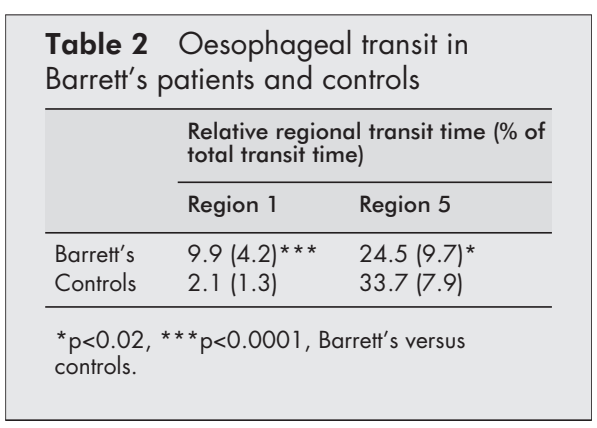

the LOS. These peaks correspond to transit times which are approximately two times longer than the average transit time and more than 10 times longer than transit in the cervicoproximal part of the oesophagus. Thus relative local transit times provide a concise description of the local kinetics of bolus transit with a longitudinal resolution of about $2.5 \%$ of the oesophagus.

A multiple swallow technique ${ }^{13}$ avoids misleading effects of single outlying swallows: the profile of summation of 180 images obtained from five swallows (fig $3 \mathrm{~B}$ ) has a shape quite similar to that of the profile of the single swallow presented in fig 3A. However, the peak maximum located near the LOS is now distinctly more prominent. Apparently, the point where relative transit time reaches a maximum in the majority of swallows is located nearer the LOS.

\section{Quantitative parameters of regional bolus transit}

The apparent band structure of the profile provides the means for quantitative evaluation of the kinetics of oesophageal bolus transport. The results of curve fitting disclose that the profile is composed of bands with a shape characteristic of a Gaussian normal distribution. The profiles of all images of five swallows obtained from a control subject can be accurately

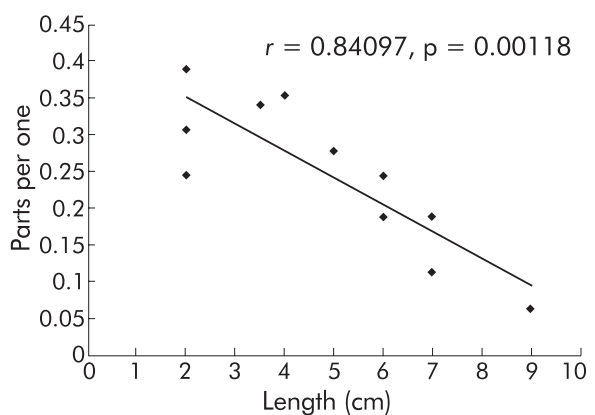

Figure 5 Correlation between the relative regional transit times of region 5 (region of the ampulla) and the length of Barrett's oesophagus in 11 Barrett's patients. The $\mathrm{x}$ axis shows the length of Barrett's oesophagus. The y axis shows the relative transit time in region 5 (ampullary region) expressed as fraction of the total oesophageal transit time (part per one). For interindividual comparisons, the length of region 5 was standardised in relation to individual oesophageal length; this length was defined by the ratio of the number of lines of the scinitgraphic array (compare with fig 2) of individuals to the average of corresponding lines of all individuals of the group.

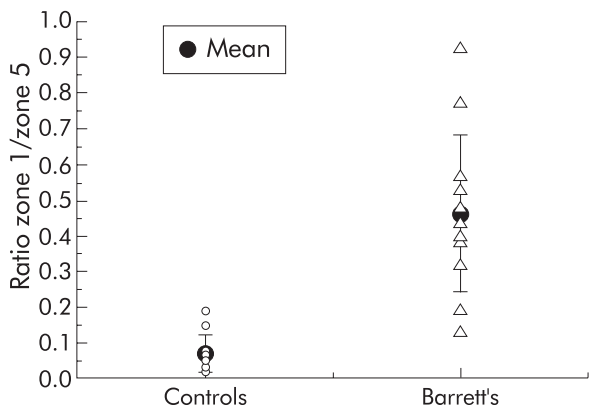

Figure 6 The ratios of relative regional transit times for region 1 (cervicoproximal region) and region 5 (region of ampulla ahead of the lower oesophageal sphincter) in 11 Barrett's patients and 12 control subjects.

fitted by superimposition of seven Gaussian bands (fig 4). The integrals of these bands represent numerical values for a quantitative description of bolus transport by their allocation to five oesophageal regions of equal length $(20 \%$ of the length of the oesophagus).

\section{Oesophageal clearance}

We compared overall oesophageal clearance in control and Barrett's patients using previously published condensed image techniques in the multiple swallow version. ${ }^{13}$ There were no significant differences between the two groups, with respect to the average percentage clearance within 10 seconds or average clearance time (table 1).

\section{Regional transit times}

Regional transit times observed in 11 control subjects showed characteristic differences from values determined in the 12 patients with Barrett's oesophagus. Major differences appeared in regions 1 (near the UOS) and 5 (the region of the ampulla) (table 2). The average of allocated integrals of Barrett's patients in region 1 was about five times larger than the corresponding average of integrals of the control group $(p<0.0001)$. In contrast, the average of the integrals for Barrett's patients in region 5 was 1.4 times smaller than the average of controls $(\mathrm{p}<0.02)$. Obviously, compared with controls, the relative time needed for bolus transit in Barrett's oesophagus patients is considerably longer in the cervical part of the oesophagus and shorter in its distal region. Interestingly, an inverse correlation between individual fractional 
transit values in the distal region (region 5) and the length of Barrett's oesophagus was observed in Barrett's patients (Pearson $=0.841 ; \mathrm{p}=0.00118$ ) (fig 5). A combination of the abnormalities observed in Barrett's disease in regions 1 and 5, obtained by dividing the average value of region 1 by the average value of region 5, gives a factor which is 6.75 times larger in Barrett's disease patients than in controls $(\mathrm{p}<0.0001)$. Overlap of the individual factors between the two groups was small (fig 6). Thus in spite of the small number of individuals investigated, discrimination was high. Altogether, these results demonstrated a significant alteration in bolus transit in Barrett's disease in both ends of the oesophagus. Apparently, deglutition as well as clearance of the bolus mass are affected.

\section{DISCUSSION}

The most important result of our oesophageal transit study and a finding which has not been reported previously was the well documented description of spatiotemporal transport pattern alterations in Barrett's oesophagus compared with controls. These alterations were seen as a significant retardation of bolus deglutition and significantly decreased accumulation in the ampullary region. These findings were made possible by an examination technique based on the concept of regional transit time topography and by development of methods for (a) image processing which visualise a profile of this topography and (b) through logic data evaluation by fitting Gaussian bands to the profile for expression of quantitative pathological indices.

The major aim of previous studies using scintigraphy for the detection of anomalous oesophageal function in Barrett's patients was limited to obtaining information on the overall clearance of the oesophagus, with inconclusive results. ${ }^{89}$ In our investigation, no difference in overall clearance between 12 Barrett's patients and 11 controls using an established technique $^{13}$ was observed. Our concept of "relative regional transit times" allows investigation of the functional elements of bolus transport in close association with oesophageal topography. Transit time profiles showed that the imaging technique we developed can characterise a two dimensional spatiotemporal pattern of bolus transport which is mechanistically related to the different regional functions of the oesophagus. In healthy subjects, transit in the proximal region of the oesophagus is much faster compared with other regions, due to forceful injection of the bolus from the pharynx. Considered in a teleological manner, this seems to be useful for protection of the airway by minimising the time of its crossing. In contrast, the bolus accumulates at the lower end in a functional ampulla before transfer of its collected mass. This diminishes the chance of gastro-oesophageal reflux. The increase in transit time in the middle region, the transitional zone of striated to smooth muscle, is related to the coupling of peristaltics of the lower to peristaltics of the upper oesophagus.

The profiles of spatiotemporal bolus distribution opened the way, through their reconstruction by superimposition of Gaussian bands, for a functional analysis of the data. ${ }^{14}$ Functional analysis represents the data in ways that aid further analysis and displays the data so as to highlight various characteristics of the investigated object. In our case, the mathematical function of Gaussian probability distribution simulates the spatiotemporal profile and "highlights" the dynamic structures of the bolus flow. A Gaussian profile results from compression of the scintigraphic image of a spheroidal or ellipsoidal body. These bodies are transient dynamic structures of the bolus mass, as shown by computer based modelling (unpublished results). Thus Gaussian bands represent structural elements of oesophageal transport. Their integrals and local position along the oesophagus are quantitative parameters that define the relative regional time the bolus needs for its transit through a given region of the oesophagus. Their allocation to different regions of the oesophagus yields numerical parameters (relative regional transit times) which have been used successfully to differentiate transport patterns of Barrett's patients from healthy controls.

Analysis of relative regional transit times of bolus transport disclosed significant differences between Barrett's patients and controls in the proximal and distal oesophagus. Compared with controls, transit time in the proximal region was greatly increased in Barrett's patients. Regarding the mechanism of oropharyngeal function in deglutition, ${ }^{10}$ two points can be made: (1) reduction in the power of the pharyngeal pump may increase the relative local transit time in the proximal oesophagus; and (2) defects in its barrier function may make the UOS leaky (for example, by weakening of the "grabbing effect"). ${ }^{15}$ Relevant to this point is our observation that release of bolus residues into the hypopharynx between swallows could be identified in five of 12 Barrett's patients. There is increasing evidence that incompetence of the UOS may cause respiratory and laryngeal symptoms in patients suffering from gastrooesophageal reflux. In one study, the ratio of proximal (hypopharynx) to distal (LOS) reflux episodes was found to be increased..$^{16}$ Decreased power of the pharyngeal pump and an incompetent UOS observed in our Barrett's patients may be pathophysiologically related. Both of these factors may contribute to the increase in the relative regional transit time in the proximal region of the oesophagus.

The observed decrease in transit time in the distal oesophagus in our Barrett's patients may be related to the role of the transient ampulla in oesophageal clearance. Emptying of the ampulla is considered to be driven by a hydrostatic pressure gradient from the oesophagus to the stomach and restoration of oesophageal length rather than by continuation of the peristaltic wave of the tubular oesophagus. ${ }^{17}$ Hence the degree of filling of the ampulla may influence its rate of emptying. Thus in Barrett's patients, reduced filling due to slower bolus progression within the upper oesophagus may prolong emptying of the ampulla. In addition, the hiatal hernia, which is frequently associated with Barrett's disease, may contribute to this delay by biphasic timing of emptying due to reflux. ${ }^{18}$ Another possible explanation for the observed decreased transit time in the distal oesophagus in Barrett's oesophagus may be related to body position during imaging. In the present study, images were obtained in the sitting posture. Although sitting is clearly the physiological posture of eating, demonstration of impaired transit in the distal oesophagus can probably better be demonstrated in the supine position because this more directly challenges the transport mechanism. Imaging in the supine position may therefore have resulted in findings that more closely matched those of studies assessing bolus propulsion with combined manometry and radiography.

In summary, the tools for processing scintigraphic images and data analysis which we developed for quantitative evaluation of the "topography of relative transit times" of oesophageal bolus transport suggest wider application of this non-invasive and comfortable technique, in particular in view of the recent considerable increase in the prevalence of Barrett associated cancer: the pathological indices obtained allow discrimination and monitoring of bolus transport alterations in Barrett's disease.

\section{ACKNOWLEDGEMENT}

The study was supported by a grant from the Association for International Cancer Research (AICR), St Andrews, UK (reference No $1 / 160)$.

Authors' affiliations

A W Stier, H J Stein, M Feith, Department of General Surgery, Klinikum rechts der Isar, TU Muenchen, Munich, Germany 
H-D Allescher, Department of Gastroenterology, Klinikum rechts der Isar, TU Muenchen, Munich, Germany

M Schwaiger, Department of Nuclear Medicine, Klinikum rechts der Isar,

TU Muenchen, Munich, Germany

\section{REFERENCES}

1 Stein HJ, Siewert JR. Barrett's esophagus, epidemiology, functional abnormalities, malignant degeneration and surgical treatment. Dysphagia 1993:8:276-88.

2 Mason RJ, Bremner CC. Motility differences between long-segment and short- segment Barrett's oesophagus. Am J Surg 1993;165:686-9.

3 Russell CO, Bright N, Buthpitiya G, et al. Oesophageal propulsive force and its relation to manometric pressure. Gut 1992;33:727-32.

4 Edmundowicz SA, Clouse RE. Shortening of the oesophagus in response to swallowing. Am J Physiol 1991;260:G512-16.

5 Pouderoux P, Lin S, Kahrilas PJ. Timing, propagation, coordination, and effect of esophageal shortening during peristalsis. Gastroenterology 1997;1 12:1147-54

6 Klein H. Esophageal transit scintigraphy. Semin Nucl Med 1995:25:306-17.

7 Kahrilas PJ, Dodds WJ, Hogan WJ. Effect of peristaltic dysfunction on esophageal volume clearance. Gastroenterology 1988;94:73-80.

8 Ham HR, Georges B, Guillaume M, et al. Evaluation of methods for qualitative and quantitative assessment of esophageal transit of liquid. Eur J Nucl Med 1985;11:17-21.
9 Karvelis KC, Drane W, Johnson DA, et al. Barrett esophagus: decreased esophageal clearance shown by radionuclide esophageal scinitgraphy. Radiology 1987; 162:97-9.

10 Conklin JL, Christensen J. Neuromuscular control of the oropharynx and oesophagus in health and disease. Annu Rev Med 1994;45:13-22.

11 Svedberg JB. The bolus transport diagram: a functional display method applied to oesophageal studies. Clin Phys Physiol Meas 1982;3:267-72.

12 Klein HA, Wald, A. Computer analysis of radionuclide esophageal transit studies. J Nucl Med 1984;25:957-94.

13 Tatsch K, Schroettle W, Kirsch CM. Multiple swallow test for the quantitative and qualitative evaluation of esophageal motility disorders. $J$ Nucl Med 1991;32:1365-70

14 Ramsay JO, Silverman BW. Functional data analysis. New York: Springer, 1997.

15 Pouderoux $\mathbf{P}$, Kahrilas PJ. Function of upper esophageal sphincter during swallowing: the grabbing effect. Am J Physiol 1997;272:G105763.

16 Shaker R, Milbrath M, Ren J, et al. Esophagopharyngeal distribution of refluxed gastric acid in patients with reflux laryngitis. Gastroenterology 1995; 109:1575-82.

17 Lin S, Brasseur JG, Pouderoux P, et al. The phrenic ampulla: distal oesophagus or potential hiatal hernia? Am J Physiol 1995;268:G320-7.

18 Mittal RK, Lange R, McCallum RW. Identification and mechanism of delayed esophageal acid clearance in subjects with hiatus hernia. Gastroenterology 1987;92:130-5. 